\title{
Avaliação das Exigências Técnicas às Exportações Brasileiras de Alimentos por Árvores de Classificação Multivariadas ${ }^{\star}$
}

\author{
- Rosane Nunes Faria* - César Augusto Taconeli**

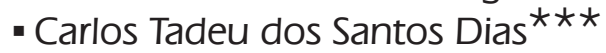

\begin{abstract}
Resumo
O objetivo deste artigo é identificar quais são as características de uma empresa que podem influenciar na dificuldade de adequação às exigências técnicas para exportação. Foi conduzido um levantamento de dados com 117 empresas exportadoras de alimentos e, como alternativa de análise dos dados, empregaram-se árvores de classificação multivariadas. Verificou-se que a característica com maior influência na dificuldade de adequação é a categoria de produto exportado, sendo as exportadoras de "Carnes e peixes frescos ou processados" as que percebem maior dificuldade. O tamanho da empresa, a adoção de normas internacionais e a experiência exportadora influenciam a dificuldade de adequação. Micro e pequenas empresas que adotam normas internacionais têm mais dificuldade para adequar seus produtos e processos às exigências técnicas que as grandes empresas. A origem do capital controlador da empresa não parece ser uma variável importante na explicação da dificuldade de adequação.
\end{abstract}

\section{Palavras-Chave}

exigências técnicas, exportação de produtos alimentares, firmas, árvores de classificação multivariadas

\begin{abstract}
The aim of this article is to identify what are the characteristics of the firm that might influence the difficulty of meeting technical requirements. It was conducted a survey with 117 firms and the data were analyzed using multivariate classification trees. It was verified that the most influential characteristic on the difficulty to meet technical requirements is the type of export product and the firms that export "fresh and processed meat and fish" are those that realize the greatest difficulty. The size of the firm, the adoption of international standards and exporting experience also influence the difficulty of adjustment. Micro and small firms that adopt international standards have more difficulties to adjust their products and process to technical requirements than larger firms. The origin of the capital is not relevant to explain the difficulty of adjustment.
\end{abstract}

\section{Keywords}

technical requirements, food products exports, firms, multivariate classification trees

\section{JEL Classification \\ F10, C14}

\footnotetext{
+ Os autores agradecem à CAPES e ao CNPO pelo financiamento parcial desta pesquisa e às empresas que colaboraram no fornecimento de informações.

* Departamento de Economia, Universidade Federal de São Carlos (UFScar) - Campus Sorocaba. E-mail: rnfaria@ufscar.br.

** Departamento de Estatística, Universidade Federal do Paraná (UFPR). E-mail: taconeli@ufpr.br.

*** Departamento de Ciências Exatas, Universidade de São Paulo, Escola Superior de Agricultura Luiz de Queiróz (ESALQ-USP). E-mail: ctsdias@esalq.usp.br.

Endereço para contato: Rod. João Leme dos Santos, Km 110 - SP-264 - Bairro do Itinga - Sorocaba - SP. CEP: 18052-780.

(Recebido em abril de 2009. Revisões requeridas em outubro de 2009. Aceito para publicação em maio de 2010).
} 


\section{Introdução}

O êxito das várias rodadas de negociações internacionais em termos de redução das barreiras tradicionais ao comércio, tais como tarifas, quotas e subsídios, tem levado os países a adotarem novos tipos de medidas protecionistas, com destaque para as normas, regulamentos e avaliação da conformidade ${ }^{1}$ (MASKUS; WILSON; OTSUKI, 2000; BEGHIN; BUREAU, 2001; VANCAUTEREN, 2002; POPER et al., 2004).

As normas e os regulamentos técnicos podem ser exigências impostas no processo de produção do produto, nas características que o produto deve apresentar, na embalagem, na rotulagem, entre outros. Para Quan (2005), as normas e os regulamentos técnicos relacionados à segurança do produto se mostram cada vez mais restritivos, principalmente aqueles referentes a alimentos, produtos farmacêuticos, cosméticos, dispositivos elétricos, brinquedos e materiais para a indústria de construção civil.

Particularmente para os consumidores, o estabelecimento de normas e regulamentos técnicos desempenha papel positivo, pois visa garantir ao consumidor produtos mais seguros, de maior qualidade, com menor impacto no meio ambiente, etc. As normas e os regulamentos também podem facilitar as transações comerciais, principalmente quando reduzem assimetria de informação entre produtor e consumidor.

Por outro lado, a existência de diferenças "naturais" entre os países, como a cultura, o nível de renda, o desenvolvimento tecnológico, entre outras, contribui para o estabelecimento de normas e regulamentos distintos entre eles. Isto representa desafios para as empresas se inserirem no comércio internacional, uma vez que elas precisam se adequar a requisitos distintos daqueles adotados em seu próprio país. Em outras palavras, a intensificação do uso de medidas técnicas pelos países compradores pode implicar custos adicionais de adequação para as empresas exportadoras.

Chen, Otsuki e Wilson (2006) argumentam que os custos de adequação incorridos pelas empresas variam de acordo com características específicas da empresa, como, por exemplo, a habilidade de adequação. Portanto, o presente estudo tem como objetivo identificar quais são as características da empresa que influenciam na dificuldade de adequação às medidas técnicas.

A escassez de informações sistematizadas sobre normas e regulamentos técnicos, para a condução de análises empíricas, sugere a necessidade de coleta de dados pri-

1 O presente estudo considera, como medidas ou exigência técnicas, as normas, os regulamentos e os procedimentos de avaliação da conformidade. 
mários por meio da aplicação de questionários. No Brasil, as informações, em nível de empresas, disponíveis atualmente, encontram-se nos trabalhos de Ferraz Filho (1997) e de Burnquist et al. (2007), porém, nenhum dos dois tem foco nos produtos alimentares. Portanto, para a presente pesquisa, foi conduzido um levantamento de dados com 117 empresas exportadoras de alimentos para levantar informações sobre as medidas técnicas enfrentadas por essas empresas.

Levantamentos de dados econômicos, realizados mediante aplicação de questionários, são invariavelmente responsáveis pela produção de grandes bancos de dados, contemplando múltiplas variáveis (qualitativas e quantitativas) e inviabilizando a aplicação das técnicas convencionais de modelagem. A aplicação de técnicas mais flexíveis de modelagem estatística torna-se fundamental na análise destes dados.

Como alternativa, emprega-se, neste estudo, árvores de classificação multivariadas, propostas por Taconeli et al. (2009), uma vez que essas possibilitam a modelagem de múltiplas variáveis qualitativas conjuntamente, com base nos resultados de um conjunto de variáveis explicativas, surgindo como uma poderosa ferramenta aplicada à análise de dados econômicos advindos de questionários.

$\mathrm{O}$ artigo visa contribuir para a literatura brasileira, tendo em vista a importância do tema para o comércio internacional e a escassez de estudos no País. O trabalho apresenta-se como uma base de conhecimento importante para pesquisadores e formuladores de políticas que pretendem trabalhar com exigências técnicas. Existe ainda uma contribuição em termos da abordagem estatística empregada para a análise dos dados. Dentre os trabalhos revisados na literatura, não foi verificado o emprego de árvores de classificação e regressão para a análise dos dados oriundos dos levantamentos sobre medidas técnicas.

O artigo está dividido em cinco seções, além desta introdução. A seção 2 apresenta a discussão teórica que embasa o trabalho e apresenta o modelo das relações empíricas que é analisado. A seção 3, por sua vez, descreve a construção de árvores de classificação e regressão nas versões uni e multivariada. A seção 4 discute o levantamento de dados e as variáveis analisadas. A seção 5 apresenta e discute os resultados encontrados e, na última seção, é feita uma breve conclusão do trabalho com algumas implicações tanto para pesquisadores quanto para tomadores de decisão.

\section{Efeitos Econômicos das Medidas Técnicas}

A principal razão para a introdução de medidas técnicas é a correção de falhas de mercado que podem surgir na presença de externalidades, assimetria de informação, 
custos de transação e bens públicos. Nestas situações de ineficiências do mercado, o estabelecimento de normas e regulamentos pode aumentar o bem-estar da sociedade e torna-se justificável ainda que isso crie impactos indesejáveis no comércio e nas forças competitivas do mercado (WORLD TRADE ORGANIZATION, 2005).

A despeito dos possíveis efeitos positivos, a introdução de medidas técnicas nem sempre resulta em ganhos líquidos seja para a economia que as introduz, seja para as economias impactadas por meio das relações comerciais mantidas com o país que impõe a regulamentação.

Diferentemente das barreiras tarifárias, o impacto de regulamentos técnicos sobre o comércio ocorre indiretamente, comumente via aumento de custos de adequação para o produtor. Em determinadas instâncias, os regulamentos podem impactar as decisões de consumo e, indiretamente, o volume das importações realizadas por um determinado país. Para avaliar esses efeitos, tem-se considerado adequado identificar, a princípio, o impacto sobre as condições domésticas de oferta e demanda e, consequentemente, as mudanças nas curvas de excesso de demanda interna, ou de demanda por importação e excesso de oferta interna, ou de oferta de exportação.

A representação gráfica de equilíbrio parcial é útil para visualizar os prováveis deslocamentos nas curvas de oferta e demanda e avaliar as mudanças nos preços, no consumo, na produção e no comércio, advindas da imposição de medidas técnicas. Os efeitos distributivos e líquidos decorrentes da introdução de uma medida técnica também podem ser visualizados via mudanças nos excedentes do produtor $\mathrm{e}$ do consumidor.

Para Thilmany e Barret (1997), a principal diferença entre os regulamentos técnicos e outros tipos de barreiras tradicionais, como cotas, tarifas, entre outras, é a possibilidade de deslocamentos da demanda com a imposição de regulamentos. Os autores alegam que regulamentos de rotulagem, de segurança e qualidade podem solucionar interesses do consumidor quanto à qualidade e segurança do produto. Portanto, podem, de forma benéfica, resolver problemas de informação imperfeita. A análise de equilíbrio parcial apresentada pelos autores deixa claro que, para regulamentos informativos, não apenas os resultados líquidos de bem-estar são ambíguos, mas o impacto no comércio também é ambíguo (Figura 1).

Na representação esquemática apresentada na Figura 1, os autores consideram um mundo com apenas dois países, sendo o país 1 o importador e o país 2, o exportador. O mercado internacional (diagrama do meio) de um dado bem $q$ é formado pela interação de excesso de demanda (ED1) e de oferta (EO2) que ocorrem em dois países: um país importador (País 1), cujo mercado doméstico do mesmo bem 
é representado no primeiro diagrama, e um país exportador (País 2), cujo mercado é representado no último diagrama.

Sob condições de livre comércio, prevalece o preço do mercado internacional, PA, nos dois países. No país 1 , o consumo estaria no ponto B enquanto a produção estaria no ponto $\mathrm{A}$, o que revela excesso de demanda no montante $\mathrm{B}-\mathrm{A}$. No país 2 , o consumo estaria no ponto I enquanto a produção, no ponto J, o que implica excesso de oferta. $\mathrm{O}$ volume comercializado é igual à $\mathrm{B}-\mathrm{A}=\mathrm{I}-\mathrm{J}=\mathrm{a}$.

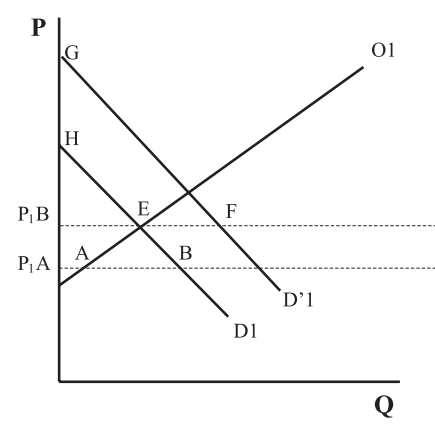

País 1 - Importador

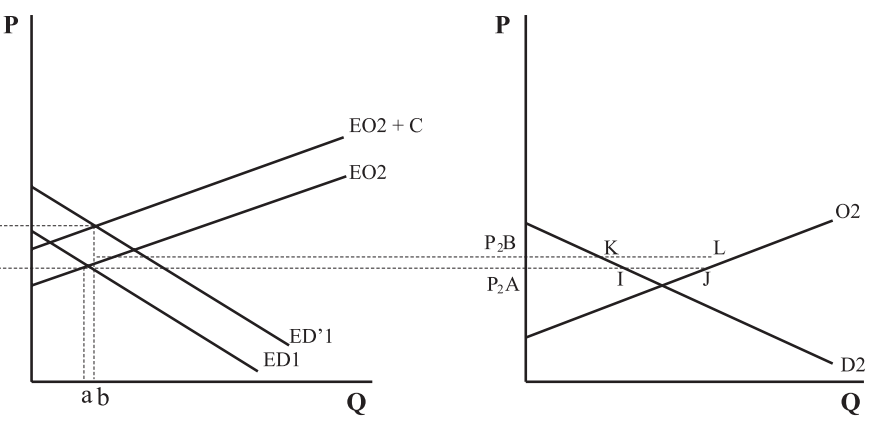

Mercado Internacional

País 2 - Exportador

Fonte: Thilmany e Barret (1997).

\section{Figura 1 - Efeitos de Regulamentos Técnicos Informativos}

Um regulamento imposto pelo país importador, por exemplo, requisitos no processo de produção ou testes na fronteira para garantir a segurança ou qualidade do produto importado, eleva os custos de exportação para o país exportador em $\mathrm{C}^{2}$ (unidades monetárias) por unidade do bem exportado, resultantes do processo de adequação à nova exigência. Portanto, a curva de excesso de oferta de exportação do país 2 se desloca de EO2 para EO2 +C. Por simplificação, dentro do país 1, assume-se que não haja deslocamento da curva de oferta com a imposição do regulamento, uma vez que os produtores domésticos já estavam adequados a uma norma e o governo a adota como um regulamento.

Caso o regulamento seja informativo para os consumidores do país importador, haverá também deslocamento da curva de demanda de Dl para D'l e, consequentemente, deslocamentos na curva de excesso de demanda de EDl para ED'l. Portanto, o novo ponto de equilíbrio no mercado internacional se dá aos preços $\mathrm{PB}$, superior ao de livre comércio, e a quantidade b também superior. No que se refere aos preços

2 Por conveniência, assume-se que o custo de adequação é constante por unidade vendida. 
no mercado internacional, o resultado é certo: sua elevação, pois sofrem pressões tanto pelo lado da oferta quanto pelo lado da demanda. Porém, para que o impacto no volume comercializado seja positivo, como mostrado na Figura 1, o regulamento precisa estimular a demanda suficientemente para compensar os aumentos de custos, caso contrário, o impacto no comércio é negativo.

No país importador, os preços se elevam de $\mathrm{P}_{1} \mathrm{~A}$ para $\mathrm{P}_{1} \mathrm{~B}$ e, como o regulamento estimula a demanda para compensar os custos, o volume importado aumenta. O bem-estar agregado aumenta somente se a área EFGH (ganho de excedente do consumidor advindo da informação) superar a área $\mathrm{ABE}$ (perda de excedente do consumidor devido aos preços mais altos).

No país exportador, considerando que o estímulo na demanda se traduz em estímulo no comércio, então os preços se elevam de $\mathrm{P}_{2} \mathrm{~A}$ para $\mathrm{P}_{2} \mathrm{~B}$, no entanto, o preço no país importador $\mathrm{P}_{1} \mathrm{~B}$ é superior ao preço no mercado exportador $\left(\mathrm{P}_{2} \mathrm{~B}\right)$ devido aos custos que o exportador precisa incorrer para adequar. $\mathrm{O}$ bem-estar agregado cresce pela área JIKL. Esse resultado tem uma implicação interessante: se o estímulo na demanda compensar os custos de adequação, o efeito no comércio é positivo e as firmas do país exportador podem ficar em uma situação melhor quando o governo do país importador impõe um regulamento.

Os vários possíveis pontos de equilíbrio que podem surgir com a imposição de regulamentos implicam ambiguidade de seus efeitos no bem-estar e no comércio. Para Thilmany e Barret (1997), a informação que o regulamento pode prover ao consumidor é o elemento-chave na determinação dos seus efeitos no comércio e bem-estar.

A despeito das ambiguidades dos impactos dos regulamentos no bem-estar e nos fluxos de comércio, os efeitos de elevação dos custos de adequação são certos e inevitáveis. A adequação às medidas técnicas envolve custos iniciais incorridos no estabelecimento de novos processos e procedimentos, como redesenho dos produtos, adaptação das unidades produtivas, desenvolvimento de infraestrutura para dar suporte aos procedimentos de avaliação da conformidade, entre outros. Envolve também custos recorrentes, que são aqueles que persistem ao longo do tempo, como, por exemplo, o uso de material mais caro para a produção destinada para algum mercado específico, operação em linhas de produção separadas para atender diferentes mercados, contratação de pessoal qualificado para os procedimentos de avaliação da conformidade (MASKUS; WILSON; OTSUKI, 2000; FRAHAN; VANCAUTEREN, 2006; POPPER, et al. 2004). 
Para Chen, Otsuki e Wilson (2006), o custo de adequação às exigências técnicas também se diferencia entre firmas com características diferentes. Os autores apresentam uma equação que mostra um componente de custo que é específico por firma. Eles supõem uma economia mundial com $\mathrm{N}$ países importadores, representados por $j=1,2,3, \ldots, \mathrm{N}$, sendo cada um desses países diferentes em termos do nível de custos fixos associados à adequação de seus requerimentos. A adequação implica custos fixos diferenciados para as firmas, denotados por:

$$
F_{i j} \equiv F_{j}+D_{i}
$$

em que $F_{j}$ é o componente comum dos custos fixos incorridos para adequar aos regulamentos impostos pelo país $j$ a um produto, ou seja, é idêntico para todos os exportadores, e $D_{i}$ é o desvio em relação a $F_{j}$, que representa os diferentes impactos que cada firma recebe dos regulamentos técnicos impostos pelo país $j$. Esse é um custo fixo específico por firma e varia conforme características da empresa, como, por exemplo, dotação tecnológica, capacidade de adequação, pessoal qualificado, entre outras. $F_{i j}$ é o custo fixo total de adequação aos regulamentos do país $j$ para um determinado produto.

Com base na discussão dos custos de adequação apresentada por Chen, Otsuki e Wilson (2006) e em outros trabalhos revisados na literatura, são apresentadas, na subseção 2.1, as relações empíricas que se pretende investigar neste artigo.

\subsection{Modelo das Relações Empíricas}

A Figura 2 apresenta algumas características da empresa que podem influenciar a sua dificuldade de adequação às exigências técnicas. A dificuldade de adequação é empregada como proxy para os custos de adequação específicos por firma, ou seja, para o componente $D_{i}$, apresentado na Equação 1. A habilidade das empresas em se ajustar às exigências técnicas pode variar dependendo do tamanho, da origem do capital controlador e da experiência exportadora da empresa. 


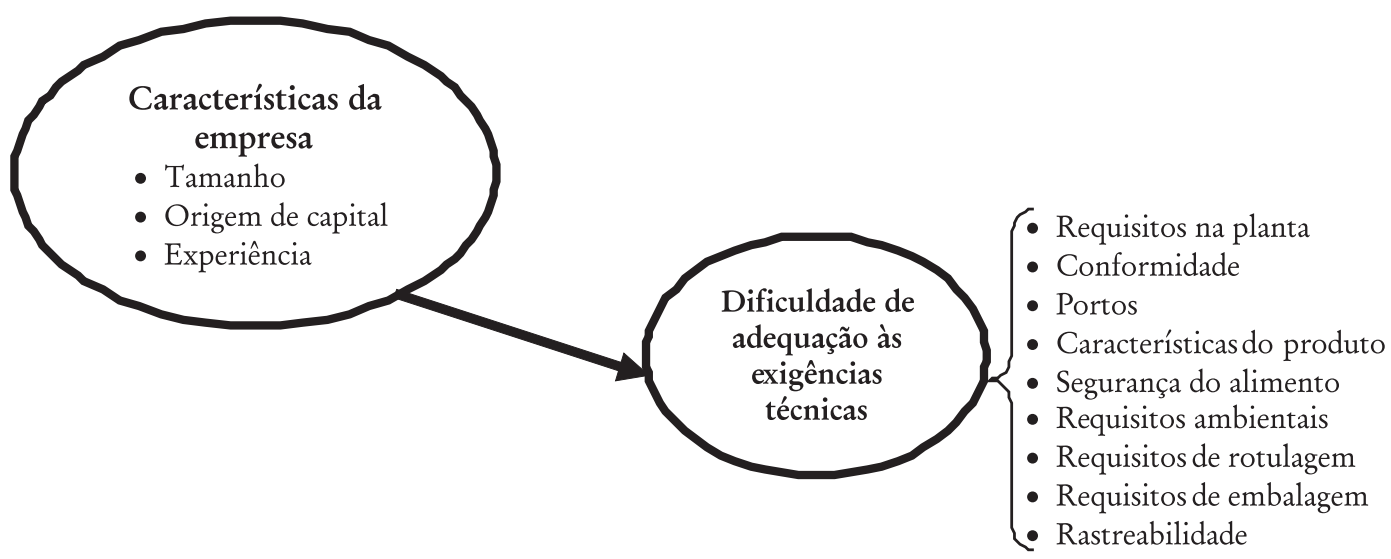

Fonte: Elaboração dos autores.

\section{Figura 2 - Modelo das Relações Empíricas}

As características da empresa foram selecionadas a partir dos resultados dos trabalhos da OCDE (2000) e Kostecki e Nowakowski (2003). O trabalho conduzido pela OCDE (2000) mostrou relação entre tamanho, tipo de capital e custos de adequação às exigências técnicas. Apesar de as empresas de todos os tamanhos terem de atender as exigências, existem vantagens para as grandes devido à possibilidade de dissolver os custos de adequação em um grande volume de vendas. As pequenas também têm maior dificuldade para se manterem atualizadas quanto às constantes mudanças nas exigências técnicas e têm menos probabilidade de ter conhecimento do mercado exterior. Portanto, essas são menos capazes de reagir rapidamente a novas oportunidades de mercado. No que se refere ao capital controlador, as multinacionais têm subsidiárias em outros países, o que facilita a obtenção de informação sobre as condições de mercado, requerimentos mandatórios e voluntários.

Para Kostecki e Nowakowski (2003), se a empresa comercializa exclusivamente no mercado doméstico, ela tem pouco interesse em obter informações a respeito de procedimentos e regras do comércio internacional. Quando a mesma percebe que as exportações podem ser uma opção atrativa, o interesse por informação sobre o mercado internacional aparece. Em um segundo estágio, a empresa se engaja em uma procura sistemática por informações sobre normas e regulamentos estrangeiros relacionados aos mercados de interesse. Em um terceiro estágio, quando ela já atua significativamente no mercado internacional, continua mantendo interesse no ambiente regulatório estrangeiro. Ou seja, as empresas com mais tempo no mercado internacional podem ter mais facilidade para obter informações e adequar as exportações às exigências técnicas. Dessa forma, a experiência exportadora também é uma característica que pode influenciar na dificuldade de adequação. 
São considerados, neste estudo, nove tipos de exigências técnicas, cuja seleção foi feita com base nos principais trabalhos revisados na literatura (ROBERTS; ORDEN; JOSLING, 1999; THORNSBURY, 1998; WILSON; OTSUKI, 2004; OCDE, 2000; BURNQUIST et al., 2007): adequação da planta e do processo de produção para a exportação; procedimentos de avaliação da conformidade do produto; requerimentos nos portos de entrada do país comprador; exigências quanto às características do produto (cor, sabor, aroma e peso); requisitos de segurança alimentar; requisitos ambientais; requisitos de rotulagem; requisitos de embalagem e requisitos de rastreabilidade do produto.

\section{3 Árvores de Classificação e Regressão}

Certos termos são utilizados de forma recorrente na caracterização dos componentes de uma árvore de regressão ou classificação. Denomina-se nó inicial à amostra original; nós intermediários, às subamostras que originam novas subamostras, e nós finais, às subamostras não partidas. Denota-se por $t$ um nó qualquer. Além disso, as partições executadas podem ser denominadas ramos. A árvore é a representação gráfica de nós e ramos. Tais termos são representados na Figura 3, que ilustra uma árvore de classificação/regressão.

As partições dos nós podem ser executadas com base em qualquer resultado amostrado das covariáveis. Seja $\left\{Y_{j}, \mathbf{X}_{j}\right\}, j=1,2, \ldots, n$ uma amostra de tamanho $n$ de uma variável dependente $Y$ e de um vetor $p$-dimensional de covariáveis $\mathbf{X}_{j}=\left(X_{1 j}, X_{2 j}, \ldots, X_{p j}\right)$. Considere $X_{l}$ uma variável ordenável (quantitativa ou qualitativa ordinal) e $\tau$ um dos resultados amostrados para $X_{l}$. Pode-se partir a amostra em duas, alocando elementos a nós distintos conforme resposta (positiva ou negativa) à questão " $X_{l j} \leq \tau$ ?" . Se $X_{l}$ for uma variável não ordenável (qualitativa nominal), os elementos são alocados a nós distintos conforme resposta (positiva ou negativa) à questão " $X_{l j} \in A$ ?", sendo $A$ um resultado (ou subconjunto de resultados) qualquer de $X_{l}$. 

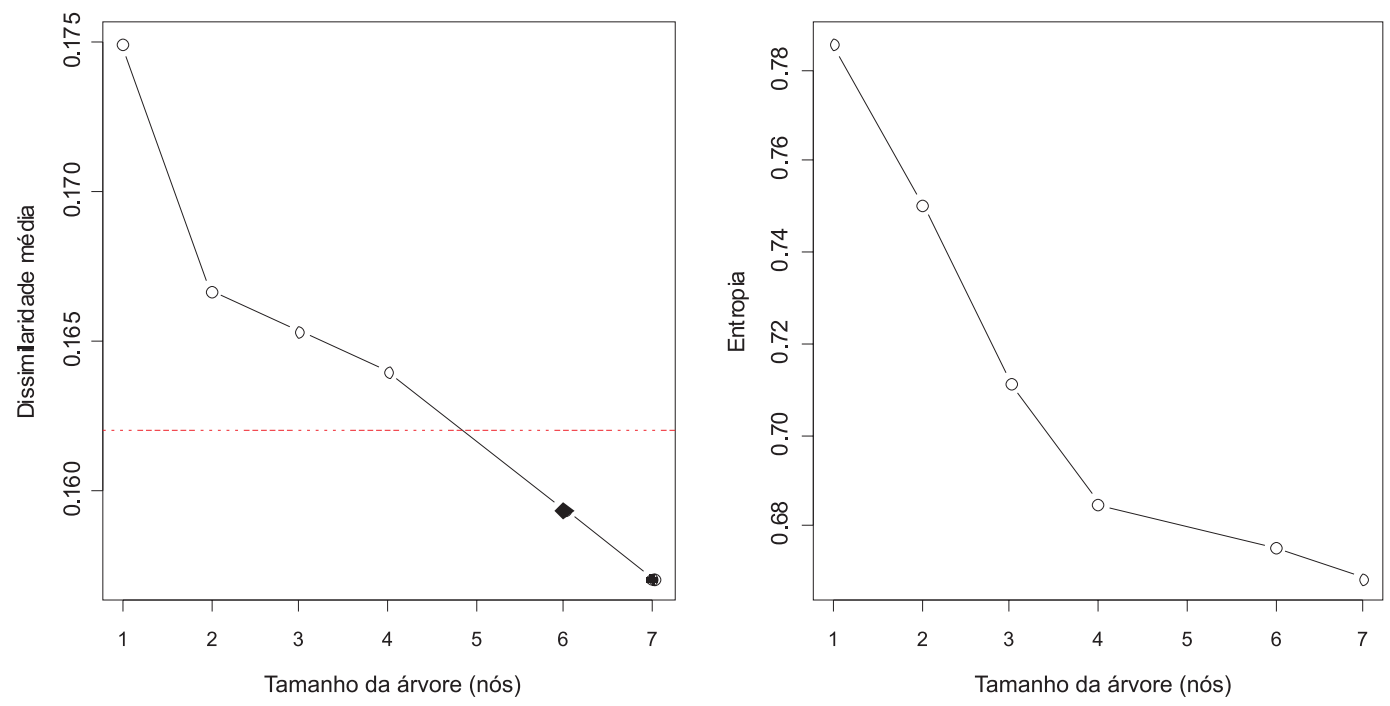

Fonte: Resultados da pesquisa.

Obs: O ponto representado por $(\bullet)$ indica a árvore com menor dissimilaridade média; o ponto representado por $(\downarrow)$, a árvore selecionada pela regra do desvio padrão, e a linha horizontal tracejada (---), o limite superior da dissimilaridade média associado à regra do desvio padrão.

\section{Figura 3 - Curvas de Custo-Complexidade para a Árvore de Classificação Multivariada Construída com Base na Dissimilaridade Média e Entropia dos Modelos}

Cada covariável, portanto, pode ser responsável por diversas partições. Deve-se executar aquela responsável por minimizar a heterogeneidade dos nós constituídos, respeitando restrições quanto ao número mínimo de elementos em nós a serem partidos e formados. Como medidas de heterogeneidade para um nó $t$, denotadas por $\phi(t)$, Breiman et al. (1984) propõem, dentre outras, o coeficiente de entropia para árvores de classificação e o índice ANOVA para árvores de regressão. Os nós devem ser partidos até a produção de uma árvore com reduzido número de elementos nos nós finais.

Construída a árvore, executa-se a poda, que tem por objetivo a obtenção de uma sequência de árvores de tamanho decrescente, cada uma das quais ótima para seu específico tamanho. A poda baseia-se na determinação de uma árvore $T$ que minimiza a seguinte função de custo-complexidade: $R_{\alpha}(T)=R(T)+\alpha|\tilde{T}|$, sendo $|\tilde{T}|$ o número de nós finais de $T ; R(T)=\sum_{t \subset T}\left(n_{t} / n\right) \phi(t)$, seu custo de má classificação; $n_{t}$ é o número de elementos em $t$, e $\alpha \geq 0$, uma constante denominada parâmetro de 
complexidade. A obtenção da sequência de árvores de tamanhos decrescentes se dá definindo a árvore $T$ que minimiza $R_{\alpha}(T)$ para uma sequência crescente de valores de $\alpha$. Dentre essas árvores, uma será selecionada mediante comparação de seus respectivos custos de más classificações.

Recomenda-se a estimação das taxas de más classificações por meio de validação cruzada e a seleção do modelo com base nas estimativas produzidas. Breiman et al. (1984) propõem a seleção da menor árvore responsável por uma taxa de erro estimada que esteja a menos de um desvio padrão, relativo ao estimador do custo adotado, da menor taxa de erro verificada.

A classificação dos nós finais de árvores de classificação e regressão tem por objetivo caracterizar cada um dos nós constituídos e possibilitar a predição de novas observações. Em árvores de classificação, os nós são classificados pela categoria da variável reposta que aparece com maior frequência, e em árvores de regressão pela média calculada entre os elementos de cada nó.

\section{1 Árvores de Classificação Multivariadas}

Árvores de classificação e regressão multivariadas permitem analisar conjuntamente múltiplas variáveis resposta mediante produção (e, consequentemente, interpretação) de um único modelo. A utilização de uma abordagem multivariada possibilita contemplar as correlações inerentes às variáveis aleatórias consideradas. Uma grande quantidade de estudos tem surgido nas últimas décadas apresentando extensões do algoritmo de classificação e regressão por árvores, direcionados à análise de dados multivariados com diferentes estruturas.

No presente trabalho, para analisar a influência das características da empresa na dificuldade de adequação às exigências técnicas, é necessária uma abordagem conjunta de nove variáveis dependentes categóricas (dificuldade de adequação às exigências técnicas) com a eminência de substanciais associações entre as mesmas e interações entre as covariáveis (características das empresas). Para esse tipo de dados e relações, as árvores de classificação multivariadas se destacam por sua flexibilidade, não impondo quaisquer restrições quanto à natureza e à distribuição das variáveis e, por sua simplicidade, tanto em relação à construção do modelo quanto à interpretação dos resultados.

Segal (1992) propõe procedimentos de regressão por árvores para dados longitudinais, fundamentando a segmentação dos nós na estrutura de médias ou de covariâncias. A construção de árvores de classificação para respostas binárias múltiplas é 
objeto de estudo de Zhang (1998), que considera, como critérios de partição das amostras, estatísticas baseadas nas matrizes de covariâncias amostrais, além de uma proposta paramétrica fundamentada em medida de entropia. Orsenigo e Vercellis (2003) propõem uma alternativa para a construção de árvores de decisão, em que cada partição é fundamentada em múltiplas variáveis, por meio de combinações lineares dos atributos considerados. Um procedimento de classificação e regressão por árvores, capaz de acomodar diferentes tipos de variáveis usando GEE (Generalized Estimation Equations), é proposto em Lee (2005). Devido a sua flexibilidade, o autor batiza a técnica como árvore de decisão generalizada multivariada. De'Ath (2002), Miller e Franklin (2002) e Larsen e Speckman (2004) propõem e utilizam árvores de regressão multivariadas em estudos ecológicos, buscando identificar relações entre características ambientais e as abundâncias das espécies consideradas.

Taconeli et al. (2009) propõem a construção de árvores de classificação multivariadas, utilizando coeficientes de dissimilaridades e entropia. Tais coeficientes são aplicados com o objetivo de quantificar a heterogeneidade dos nós constituídos, fundamentando a seleção das partições a serem executadas e a poda, e como medida de má classificação na validação cruzada. Na sequência, são descritas medidas de heterogeneidade intranós e de erro de predição, apresentadas em Taconeli et al. (2009) e empregadas neste artigo.

Sejam $Y_{1}, Y_{2}, \ldots, Y_{q}$ variáveis aleatórias qualitativas, cada uma com $r_{i}$ possíveis resultados $(i=1,2, \ldots, q)$; a entropia do vetor aleatório $\mathbf{Y}=\left(Y_{1}, Y_{2}, \ldots, Y_{q}\right)$ é definida como:

$$
H(\mathbf{Y})=\sum_{i=1}^{q} H\left(Y_{i}\right)
$$

sendo $H\left(Y_{i}\right)=-\sum_{k=1}^{r_{i}}\left[P\left(Y_{i}=y_{k}\right)\right] \log _{2}\left[P\left(Y_{i}=y_{k}\right)\right]$. Dispondo-se apenas de uma amostra, as probabilidades requeridas são desconhecidas, devendo ser estimadas pelas respectivas frequências amostrais, ou seja,

$$
\hat{H}\left(Y_{i}\right)=-\sum_{k=1}^{r_{i}} p_{i k} \log _{2}\left[p_{i k}\right]
$$

sendo $p_{i k}$ a proporção amostral do resultado $k$ da $i$-ésima variável. Quanto menor o valor de $\hat{H}$, maior a concentração dos elementos amostrais em um pequeno nú- 
mero de categorias das variáveis sob estudo, ou seja, menor a heterogeneidade amostral. Assim, pode-se considerar, como medida de heterogeneidade de um nó $t$ $\phi_{\text {Ent }}(t)=\hat{H}_{t}(\mathbf{Y})$, a entropia multivariada calculada com base nos elementos contidos em $t$, utilizando-a como base para a execução das partições e como taxa de má classificação no processo de poda.

Coeficientes de dissimilaridades também podem ser utilizados como medida de heterogeneidade dos nós na construção do modelo. Tais coeficientes são aplicados com o objetivo de quantificar a dessemelhança entre um par de elementos com relação a um conjunto de atributos. Quanto maior o valor do coeficiente, maior a discrepância entre os elementos considerados. Taconeli et al. (2009) apresentam diferentes coeficientes de dissimilaridades, discutindo suas propriedades e aplicando-os na construção de árvores de classificação multivariadas. Dentre eles, o coeficiente de dissimilaridades simples consiste basicamente na proporção de atributos não coincidentes. Goodall (1966) apresenta um coeficiente de dissimilaridades que incorpora as frequências amostrais dos atributos, ponderando mais fortemente atributos menos frequentes. Já o coeficiente proposto em Quang e Bao (2005) baseia-se em distribuições de probabilidades condicionais. A utilização de diferentes coeficientes pode, ocasionalmente, produzir diferentes modelos, dadas as diferentes propriedades apresentadas pelos mesmos.

Para uma medida de dissimilaridade $D$ qualquer, seja $D_{j j^{\prime}}$ a dissimilaridade calculada para dois elementos $j$ e $j^{\prime}$, aplica-se como medida de heterogeneidade para $n$ elementos que constituem um nó $t$ a dissimilaridade média entre tais elementos, ou seja,

$$
\varphi_{D i s}(t)=\left[\frac{n(n-1)}{2}\right]^{-1} \sum_{j=1}^{n} \sum_{j^{\prime}<j^{\prime}} D_{j j^{\prime}}
$$

Para o processo de seleção do modelo, Taconeli et al. (2009) propõem o uso de coeficientes de dissimilaridades. Propõem ainda, como medida de qualidade preditiva para uma nova observação $y^{*}$, independente daquelas utilizadas na construção da árvore e alocadas, segundo o modelo, a um nó $t$, a dissimilaridade média de $y^{*}$ com relação aos elementos que constituem o referido nó, ou seja,

$$
\phi_{D i s}\left(y^{*}\right)=\sum_{j \subset t} d_{j}^{*} / n_{t}
$$

sendo $d_{j}^{*}$ a dissimilaridade de $y^{*}$ com relação a um elemento $j \subset t$. 
A interpretação dos resultados produzidos por árvores de classificação e regressão multivariadas é facilitada mediante execução de procedimentos exploratórios multivariados. Para árvores de classificação com múltiplas respostas, Taconeli et al. (2009) propõem a utilização de análise de correspondência múltipla (GREENACRE, 1984; RENCHER, 2001), técnica multivariada capaz de explorar a associação entre múltiplas variáveis categorizadas por meio da representação de suas categorias por pontos no espaço Euclideano. Nessa representação, proximidade entre pontos que representam categorias de diferentes variáveis indica forte associação entre as mesmas, enquanto que a proximidade entre pontos que representam categorias de uma mesma variável indica que tais categorias poderiam ser combinadas em uma só, dada a semelhança entre indivíduos que compõem essas duas categorias com relação aos resultados das demais variáveis. A produção de gráficos de análise de correspondência permite, portanto, investigar visualmente as associações existentes num conjunto de variáveis qualitativas.

O software estatístico R (R DEVELOPMENT CORE TEAM, 2009) foi utilizado na programação e execução dos algoritmos - apresentados na íntegra em Taconeli (2008) - e na produção dos gráficos que complementam os modelos.

\section{Os Dados e as Variáveis}

O instrumento de coleta de dados foi um questionário aplicado a empresas exportadoras de alimentos. Para a seleção da amostra, utilizou-se o catálogo de exportadores brasileiros disponível no sítio da Confederação Nacional das Indústrias, CNI, cujas empresas responderam, no biênio 2005/2006, por mais de $90 \%$ do valor das exportações brasileiras e $91,2 \%$ do total do número de produtos exportados. O catálogo totaliza 932 empresas com exportações de alimentos acima de US\$ 100 mil no biênio 2005/06 e contempla empresas de todos os Estados brasileiros, de diversos tamanhos e diferentes composições de capital.

O questionário estruturado, elaborado pelos autores, foi aleatoriamente enviado por e-mail para 400 empresas $^{3}$ que exportaram em 2006, sendo que, dessas, 117 os retornaram corretamente preenchidos e compõem a amostra deste trabalho. Para o envio do questionário, foi feito primeiramente um contato por telefone com o gerente, diretor ou responsável pela área de exportação para apresentar a pesquisa e coletar o e-mail do informante. O Quadro 1 apresenta cada variável utilizada neste estudo, de acordo com sua denominação e classificação quanto à escala de medida.

3 Esse foi o total de empresas que apresentaram o número do telefone correto no catálogo de exportadores da CNI e, portanto, foi possível fazer o contato telefônico. 


\begin{tabular}{|c|c|c|}
\hline Nome & Variável & Classificação \\
\hline \multicolumn{3}{|c|}{ Dificuldade de adequação às exigências técnicas } \\
\hline $\begin{array}{l}\text { DPla } \\
\text { DConf } \\
\text { DPor } \\
\text { DCar } \\
\text { Dseg } \\
\text { DAmb } \\
\text { DRot } \\
\text { DEmb } \\
\text { DRast }\end{array}$ & $\begin{array}{l}\text { Requisitos na planta e processo de produção } \\
\text { Procedimentos de avaliação da conformidade } \\
\text { Portos de entrada do país comprador } \\
\text { Características do produto } \\
\text { Requisitos de segurança do alimento } \\
\text { Requisitos ambientais } \\
\text { Requisitos de rotulagem } \\
\text { Requisitos de embalagem } \\
\text { Requisitos de rastreabilidade }\end{array}$ & $\begin{array}{l}\text { 1. Baixa Dificuldade } \\
\text { 2. Média Dificuldade } \\
\text { 3. Alta Dificuldade }\end{array}$ \\
\hline \multicolumn{3}{|c|}{ Características da empresa } \\
\hline Nfunc & Tamanho da empresa - Número de funcionários & Quantitativa \\
\hline Ocap & Origem do capital controlador & $\begin{array}{l}\text { Qualitativa Nominal } \\
\text { 1. Nacional } \\
\text { 0. Não Nacional }\end{array}$ \\
\hline Tatu & $\begin{array}{l}\text { Experiência exportadora - Tempo de atuação no } \\
\text { mercado exportador }\end{array}$ & Quantitativa \\
\hline \multicolumn{3}{|c|}{ Variáveis de Controle } \\
\hline Cert & Adoção de normas internacionais & $\begin{array}{l}\text { Qualitativa Nominal } \\
\text { 1. Sim } \\
\text { 0. Não }\end{array}$ \\
\hline Dest & Dois principais mercados de destino & $\begin{array}{l}\text { Qualitativa Nominal } \\
\text { 1. País desenvolvido } \\
\text { 2. País em desenvolvimento } \\
\text { 3. Ambos }\end{array}$ \\
\hline Cat & Categoria de produtos exportados & $\begin{array}{l}\text { Qualitativa Nominal } \\
\text { 1. Carnes e peixes frescos e processados } \\
\text { 2. Frutas e vegetais frescos e processados } \\
\text { 3. Açúcares, produtos de confeitaria e } \\
\text { cacau } \\
\text { 4. Café e chá } \\
\text { 5. Bebidas } \\
\text { 6. Outros }\end{array}$ \\
\hline
\end{tabular}

\section{Quadro 1 - Denominação e Classificação das Variáveis do Modelo das Relações Empíricas}

Além das características da empresa, foram incluídas também três variáveis de controle: categoria de produtos exportados (Cat), certificação (Cert) e os mercados de destino (Dest). O controle para o tipo de produto é importante, pois exigências técnicas diferentes são impostas a produtos diferentes. 
A adoção de normas internacionais é empregada como variável de controle, pois a equação apresentada por Chen, Otsuki e Wilson (2006) considera apenas custos fixos relacionados aos regulamentos técnicos e, no questionário aplicado, foram considerados tanto regulamentos técnicos quanto normas técnicas.

Os dois principais países de destino também foram incluídos como variável de controle, pois Chen, Otsuki e Wilson (2006) preveem custos fixos de adequação de impostos por um país $j$. No entanto, no questionário não foi especificado um destino específico.

Nas árvores de classificação, foram tomadas, como variáveis resposta, as nove variáveis relativas às dificuldades de adequação às exigências técnicas e, como explicativas, as demais variáveis apresentadas no Quadro 1.

\section{Resultados e Discussão}

Um possível problema na construção de árvores de classificação e regressão é a presença de viés na seleção das variáveis responsáveis pelas partições. Variáveis com maior número de partições possíveis tendem a gerar partições com maior frequência, em detrimento a outras com menos partições possíveis, ainda que sua associação com as variáveis resposta seja mais fraca do que alguma (ou algumas) das variáveis do segundo grupo. Para eliminar possíveis vícios de seleção, Lee e Shih (2006) propõem a busca pelas partições em duas etapas: primeiramente seleciona-se a variável a produzir a partição, baseando-se em testes de independência condicional para tabelas de contingência de três entradas. Uma vez selecionada a variável, busca-se a melhor partição que ela produz. Essa técnica foi aplicada, no presente trabalho, para eliminação do viés de partição. A árvore de classificação multivariada selecionada é apresentada na Figura 4. O coeficiente multivariado de entropia, apresentado na seção 3, foi utilizado na construção, poda e seleção do modelo. A denominação das variáveis dentro dos nós está de acordo com o Quadro 1. 


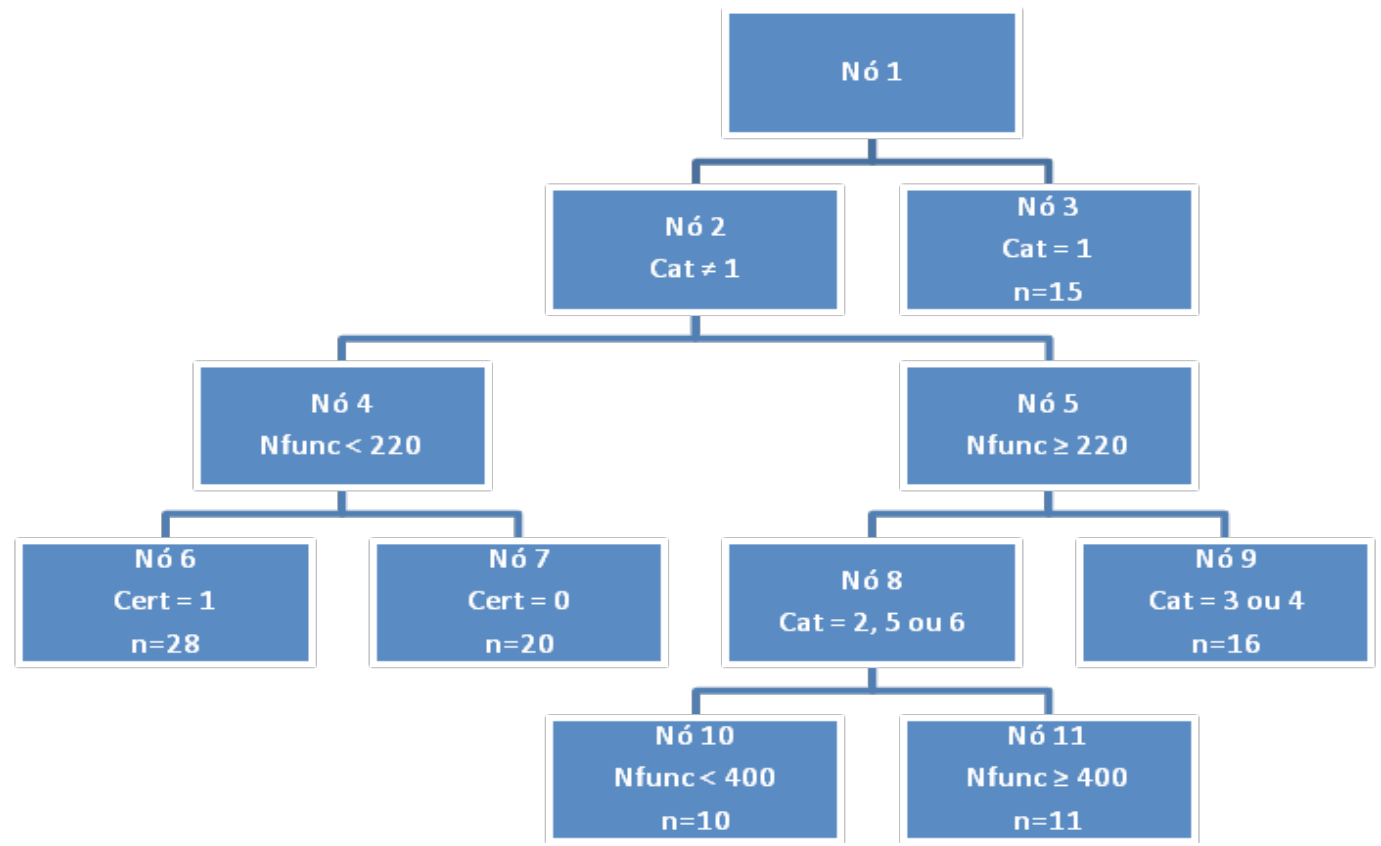

Fonte: Resultados da pesquisa.

Obs: No interior dos nós, são apresentadas as partições pelas quais estes foram formados e, nos nós finais, o número de empresas que os constituem. Os nós com preenchimento são nós finais.

\section{Figura 4 - Árvore de Classificação Multivariada}

A categoria de produtos que a empresa exporta (Cat) é a variável explanatória mais importante na explicação da percepção de dificuldade de adequação às exigências técnicas, uma vez que, além de produzir duas partições, ela é a primeira variável a ser incorporada ao modelo e é responsável pela maior redução da heterogeneidade dos nós. As outras variáveis que também compõem o modelo são: a adoção de normas internacionais (Cert), o número de funcionários (Nfunc) e o tempo de atuação no mercado exportador (Tatu). O nó 3 é composto apenas pelas empresas que exportam "Carnes e peixes frescos e processados", enquanto o nó 2 compreende empresas que exportam todas as outras categorias de produtos. Apenas a título de exemplo, o nó 6 é composto por empresas que não exportam "Carnes e peixes frescos e processados" com cinco anos ou menos de atuação no mercado exportador e que adotam normas internacionais, conforme as partições que resultaram em sua constituição.

A Figura 5 apresenta a distribuição de frequências para as dificuldades de adequação das empresas que constituem cada um dos nós finais da árvore. No eixo horizontal, estão discriminadas as dificuldades de adequação para cada um dos nove tipos de exigências técnicas e, no eixo vertical, a proporção de empresas em cada nó, que 
respondeu baixa, média ou alta dificuldade. A análise conjunta destes gráficos com a árvore de classificação multivariada (Figura 4) é determinante para a identificação das associações de interesse.
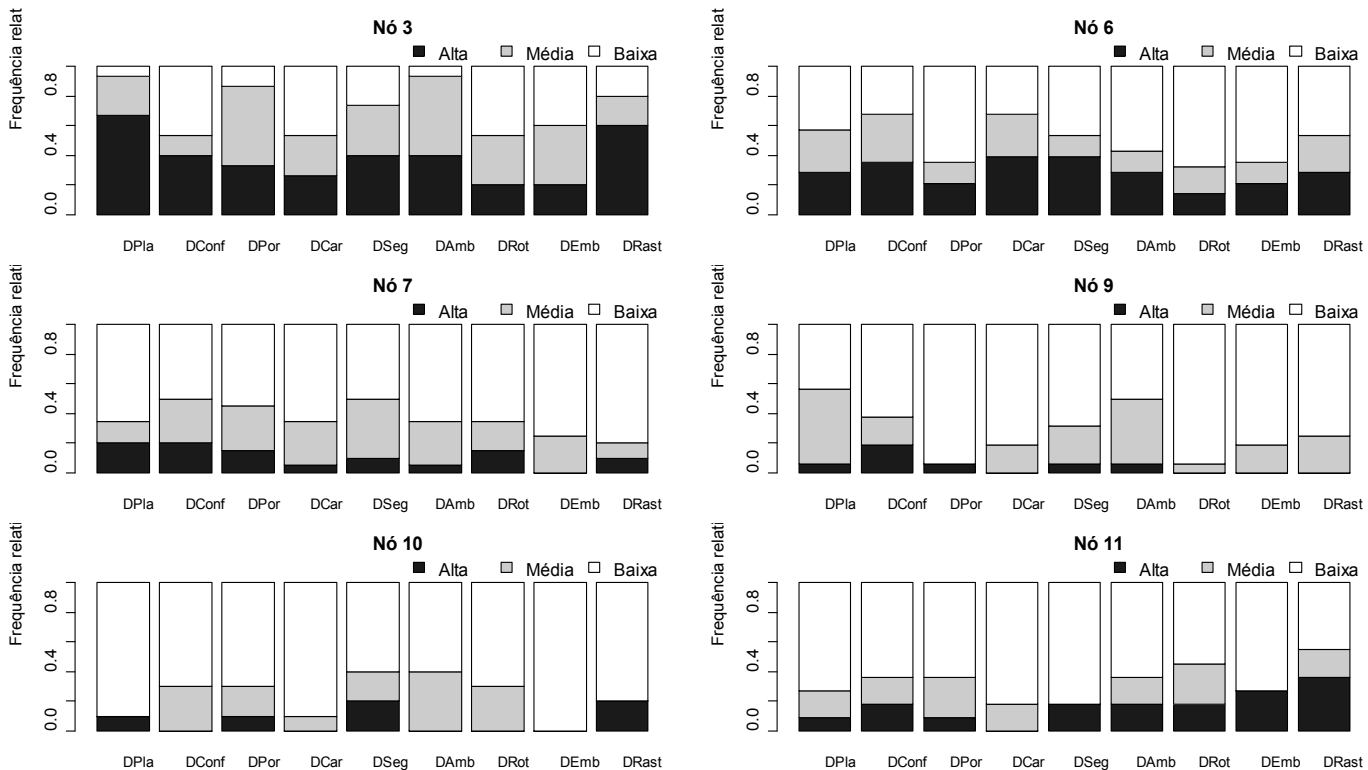

Fonte: Resultados da pesquisa.

Figura 5 - Distribuição dos Nós Finais da Árvore de Classificação Multivariada Segundo as Variáveis de Dificuldade de Adequação

A Figura 5 evidencia, inicialmente, a existência de três grupos de empresas que se diferenciam em termos de dificuldade de adequação. As empresas que constituem os nós 3 e 10 apresentam maiores dificuldades de adequação. Em seguida, destacam-se as empresas do nó 9 , que manifestam dificuldade de adequação intermediária no conjunto de exigências técnicas abordadas. Por fim, as outras empresas agrupadas nos nós 6,8 e 11 manifestam maciçamente baixa dificuldade de adequação.

O nó 3, composto pelas empresas exportadoras de "Carnes e peixes frescos e processados", se destaca como o nó em que as empresas percebem maior dificuldade de adequação para praticamente todos os tipos de exigências técnicas. Nota-se que as colunas do gráfico desse nó estão quase que inteiramente preenchidas com as cores preta e cinza escuro, indicadoras de alta e média dificuldade de adequação, respectivamente. Os requisitos na planta e processo de produção e os requisitos de 
rastreabilidade foram considerados por aproximadamente 70\% das empresas desse nó como de alta dificuldade de adequação.

Empresas com menos de 269 funcionários, ou seja, micro, pequenas e algumas médias empresas, ${ }^{4}$ que adotam normas internacionais e têm no mínimo seis anos de atuação no mercado exportador, também percebem alta dificuldade de adequação, conforme pode ser visto pela distribuição de frequências no gráfico correspondente ao nó 10. Por exemplo, a obtenção de uma certificação internacionalmente aceita implica custos iniciais e custos recorrentes como análises laboratoriais, despesas com deslocamentos de inspetores e taxas administrativas, o que pode elevar a percepção de dificuldade de adequação especialmente para as micro e pequenas empresas, que não têm recursos financeiros e pessoal qualificado. Observa-se que o nó 11 se diferencia do nó 10 pelo tamanho da empresa (Nfunc), o que evidencia que essa variável é importante para explicar a percepção de dificuldade de adequação e corrobora o fato de que as micro, pequenas e algumas médias empresas são as que manifestam maiores dificuldades.

O nó 9 é composto pelas empresas que não adotam normas internacionais e exportam basicamente produtos das categorias "Café e chá" e "Outros", totalizando $75 \%$ do total das empresas do nó. É importante observar que a categoria "Outros" engloba produtos como o leite e laticínios, ovos de aves, mel, etc., produtos da indústria da moagem e maltes, gorduras, óleos e ceras animais e vegetais, preparações de produtos hortícolas, preparações alimentícias diversas e resíduos e desperdícios das indústrias alimentícias, etc. Ou seja, o nó 9 engloba empresas que exportam produtos menos processados.

Entre as empresas que manifestam baixa dificuldade de adequação, ressaltam-se os nós 6 e 8, sendo que o primeiro é constituído por empresas que possuem cinco anos ou menos de atuação no mercado exportador e adotam normas internacionais. Uma possível explicação para esse resultado é que as empresas desse nó podem adotar, como estratégia, a construção da unidade industrial e o desenvolvimento dos produtos já voltados para atender os requisitos dos mercados compradores, uma vez que são, em sua maioria, jovens ( $70 \%$ foram fundadas na década de noventa) e mais de $50 \%$ têm foco no mercado internacional (possuem idade igual ao tempo de atuação no mercado exportador). Cabe ressaltar ainda que, mesmo com a unidade industrial adaptada às exigências técnicas, existem alguns requisitos como procedimento de avaliação de conformidade do produto e adequação da embalagem, que são cons-

4 O Serviço Brasileiro de Apoio às Micro e Pequenas Empresas - SEBRAE - adota uma classificação para o tamanho das empresas industriais de acordo com o número de pessoas ocupadas. Seguindo essa classificação, as empresas amostradas foram classificadas em Micro e Pequenas Empresas (até 99 funcionários), Médias Empresas (100 a 499 funcionários) e Grandes Empresas (acima de 500 funcionários). 
tantemente modificados e solicitados pelos países importadores e possuem custo alto. No nó 8, agrupam-se empresas que exportam produtos da categoria "Bebidas" e sucos de frutas processados, que estão classificados na categoria "Frutas e vegetais frescos e processados", ou seja, são empresas que exportam alimentos com nível de processamento superior ao das empresas do nó 9. Esse resultado parece indicar que as empresas exportadoras de produtos menos processados percebem maior dificuldade de adequação. "Carnes e peixes frescos e processados" parece ser a categoria de produtos com requisitos mais restritivos, seguida pelas categorias "Café e chá" e "Outros".

A Figura 6 apresenta o resultado produzido por uma análise de correspondência múltipla aplicada ao conjunto de variáveis resposta permitindo avaliar a associação entre os nós e as variáveis de dificuldade de adequação. Inicialmente, verifica-se a segmentação dos diferentes graus de dificuldade, indicando que empresas com elevado grau de dificuldade de adequação para uma das exigências técnicas tendem a manifestá-la também em relação às demais. $\mathrm{O}$ mesmo ocorre para empresas com médias ou baixas dificuldades. A proximidade do nó 3 e 10 às altas dificuldades de adequação, do nó 9 às altas e médias, e dos nós 6,8 e 11 às baixas, por exemplo, ratificam as conclusões anteriormente citadas.

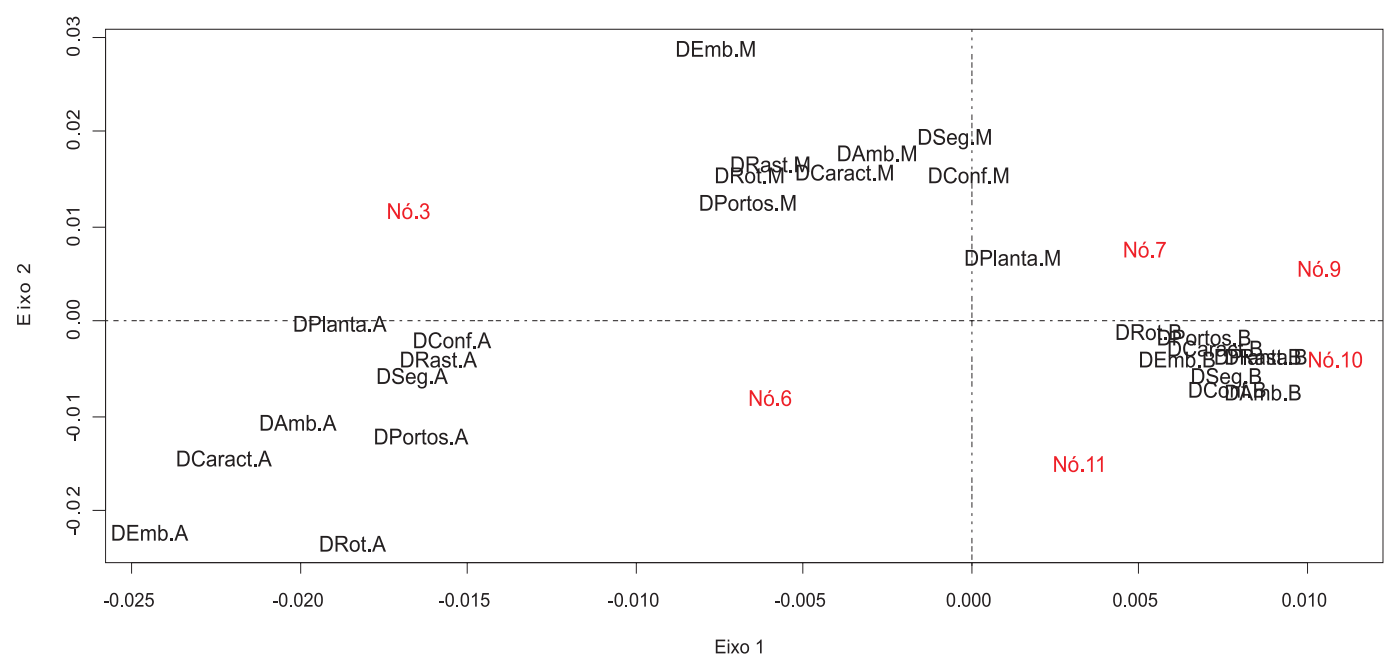

Fonte: Resultados da pesquisa.

Obs: Acompanhada de cada dificuldade, as letras 'A', 'M' e 'B' indicam, respectivamente, 'alta', 'média' e 'baixa'. Os números representados no interior do gráfico indicam os nós finais.

Figura 6 - Gráfico da Análise de Correspondência Múltipla para as Variáveis de Dificuldade de Adequação 


\section{Conclusões}

Os resultados da árvore de classificação multivariada indicaram, primeiramente, a importância da categoria de produtos que a empresa exporta na percepção de dificuldade de adequação, o que evidencia a necessidade de se considerar o tipo de produto exportado na condução de análises de exigências técnicas. Também permitiram identificar características das empresas que influenciam a dificuldade de adequação como o tamanho da empresa, a adoção de normas internacionais e a experiência exportadora. Observou-se que as micro, pequenas e algumas médias empresas que adotam normas internacionais são as que percebem maiores dificuldades. Outro resultado a ser ressaltado é a indicação de que a produtos menos processados são impostas exigências mais restritivas. Unnevehr (2000) argumenta que as questões de segurança do alimento são mais relevantes para produtos frescos ou menos processados, uma vez que esses produtos são transferidos e consumidos na forma "in natura".

A origem do capital controlador da empresa não se mostrou importante na explicação da dificuldade de adequação às exigências técnicas, o que pode ser consequência da predominância de empresas de capital nacional na indústria de alimentos no Brasil e, consequentemente, na amostra analisada neste estudo. Adicionalmente, foi apresentada a aplicabilidade de árvores de classificação multivariadas na modelagem de dados complexos de comércio internacional, em particular, e dados econômicos, em geral.

Os resultados encontrados são úteis em termos de elaboração de políticas públicas, que têm como objetivo auxiliar as empresas no cumprimento de medidas técnicas. Atenção especial deve ser dispensada principalmente às micro e pequenas empresas e também médias empresas, que demonstraram maior dificuldade de adequação às exigências técnicas. Também é de extrema importância o aperfeiçoamento dos controles sanitários e fitossanitários para os produtos menos processados, que são aqueles aos quais são impostas exigências técnicas mais restritivas.

Por fim, cabe mencionar que a amostra inclui apenas empresas que exportaram no ano de 2006, porém, podem existir empresas que têm potencial para comercializar internacionalmente e não o fazem devido aos custos de adequação às exigências técnicas. Portanto, para algumas empresas, principalmente micro e pequenas, as exigências podem ser proibitivas. 


\section{Referências}

BREIMAN, L.; FRIEDMAN, J. H.; OLSHEN, R. A.; STONE, C.J. Classification and regression trees. Califórnia City: Wadsworth International Group, 1984. $358 \mathrm{p}$.

BEGHIN, J. C.; BUREAU, J. C. Quantitative policy analysis of sanitary, phytossanitary and technical barriers to trade. Économie Internationale, Paris, v. 1, n. 87, p. 107-130, 2001.

BURNQUIST, H. L. et al. Análise de evidências sobre a importância de barreiras técnicas à exportação de empresas brasileiras. Revista de Economia Aplicada, Ribeirão Preto, v. 11, p. 209-229, 2007.

CHEN, M. X.; OTSUKI, T.; WILSON, J. S. Do standards matter for export success? World Bank Policy Research, Washington, 2006. (Working Paper n. 3809). Disponível em: <http://www-wds.worldbank.org/servlet/WDSContentServer/WDSP/IB/2006/01/18/000016406_20060118152026/Rendered/PDF/ wps3809.pdf >. Acesso em: 5 jan 2007.

DE'ATH, G. Multivariate regression trees: a new technique for modeling species-environment relationships. Ecology, Brooklin, v. 83, n. 4, p. 1105-1117, 2002.

FERRAZ FILHO, G. Barreiras técnicas ao comércio internacional: a experiência das exportações brasileiras. Revista Brasileira de Comércio Exterior, n. 52, p. 47-56, 1997.

FRAHAN, B. H; VANCAUTEREN, M. Harmonisation of food regulations and trade in the single market: evidence from disaggregated data. European Review of Agricultural Economics, v. 33, n. 3, p. 337-360, 2006.

GOODAL, D. W. A new similarity index based on probability. Biometrics, Washington, v. 22, n. 4, p. 882, 1996.

GREENACRE, M. J. Theory and applications of correspondence analysis. London: Academic Press, 1984.

KOSTECKI, M. M.; NOWAKOWSKI, M. How do managers view regulatory barriers to export of services? An empirical investigation of Poland-based firm. Neuchâte: Université de Neuchâtel, Sep. 2003. Disponível em: <http:// www.unine.ch/ gge/paper/crmm99-04.pdf - Resultado Adicional> . Acesso em: 25 fev. 2007.

LARSEN D. R., SPECKMAN P. L. Multivariate regression trees for analysis of abundance data. Biometrics, Washington, v. 60, n. 2, p. 543-549, 2004.

LEE, S. K. On generalized multivariate decision tree by using GEE. Computational Statistics \& Data Analysis, Amsterdan, v. 49, n. 4, p. 1105-1119, 2005.

LEE, T. H.; SHIH, Y. S. Unbiased variable selection for classification trees with multivariate response. Computational Statistics \& Data Analysis, Amsterdan, v. 51, p. 659-667, 2006. 
MASKUS, K. E.; WILSON, J. S; OTSUKI, T. Quantifying the impact of technical barriers to trade. A framework for analysis. The World Bank Development Research Group, Dec. 2000. 56p. (Policy Research Working Paper 2512).

MILLER, J.; FRANKLIN, J. Modeling the distribution of four vegetation alliances using generalized linear models and classification trees with spatial dependence. Ecological Modelling, Amsterdan, v. 157, n. 2-3, p. 227-247, 2002.

OCDE. An assessment of the costs for international trade in meeting regulatory requirements. Paris: Organization for Economic Co-operation and Developement. (Working Party of the Trade Committee 2000). Disponível em: <http://www. oecd.org/ech/>. Acesso em: 8 set. 2008.

ORSENIGO, C.; VERCELLIS, C. Multivariate classification trees based on minimum features discrete support vector machines. Journal of Management Mathematics, Nova York, v. 14, p. 221-234, 2003.

POPPER, S. W.; GREENFIELD, V. ; CRANE,K.; MALIK, R. Measuring economic effects technical barriers to trade on U.S exporters. Gaithersburg: Md. National Institute of Standards and Technology, 2004. Disponível em: <http://www. google.com/search?hl=pt-BR\&lr=\&q=\%22Popper $\% 22+\% 22 \mathrm{Measuring}+$ Eco nomic +Effects\%22>. Acesso em: 13 nov. 2006.

QUAN, Y. 10 trends in technical barriers to trade. Xangai: China Economic Net, 2005. 4 p. Disponível em: <http://en-1.ce.cn/Insight/200512/28/ t20051228_5655736.shtml>. Acesso em: 1 fev. 2006.

QUANG, L. S.; BAO, H. T. An association-based dissimilarity measure for categorical data. Pattern Recognition Letters, Amsterdam, v. 26. p. 2549-2557, 2005.

R DEVELOPMENT CORE TEAM. R: a language and environment for statistical computing. Vienna, Austria: RProjetc.org, 2009. Disponível em: <http://www.Rproject.org >. Acesso em: 13 out. 2009.

RENCHER, A. C. Methods of multivariate analysis. New York: John Wiley, 2001. $693 p$.

ROBERTS, D.; ORDEN, D.; JOSLING, T. A framework for analysing technical barriers to agricultural markets. Washington, DC: Market and Trade Economics Division, US. Department of Agriculture, 1999. (Thechnical Bulletin, n. 1876)

SEGAL, M. R. Tree-structured methods for longitudinal data. Journal of the American Statistical Association, Boston, v. 87, p. 407-418, 1992.

TACONELI, C. A. Árvores de classificação multivariadas fundamentadas em coeficientes de dissimilaridade e entropia. 2008. 100 p. Tese (Doutorado em Estatística e Experimentação Agronômica) - Escola Superior de Agricultura "Luiz de Queiroz", Universidade de São Paulo, Piracicaba, 2008.

TACONELI, C. A.; ZOCCHI, S. S.; DIAS, C. T. S. Extensões do algoritmo de árvores de classificação para a análise de dados categorizados multivariados utilizando 
coeficientes de dissimilaridade e entropia. Revista Brasileira de Biometria, Jaboticabal, v. 27, n. 1, p. 93-114, 2009.

THILMANY, D. D.; BARRET, C. B. Regulatory barriers in an integrating world food market. Review of Agricultural Economics, v. 19, n. 1, p. 91-107, 1997.

THORNSBURY, S. Technical regulations as barriers to agricultural trade. 1998. 192 p. Dissertation (Doctor of Philosophy In Agricultural and Applied Economics) - Faculty of Virginia Polytechnic Institute and State University, Blacksburg, 1998.

UNNEVEHR, L. J. Food safety issues and fresh food product exports from LDCs. Agricultural Economics, Milwaukee, v. 23, n. 3, p. 231-240, 2000.

VANCAUTEREN, M. The impact of technical barriers to trade on home bias: an application to EU data. Bruxelles: Université Catholique de Louvain, Institut de Recherches Economiques et Sociales, Aug. 2002. 36 p. (Discussion paper, 2002032). Disponível em: < http://www.ires.ucl.ac.be/DP/IRES_DP/2002-32. pdf>. Acesso em: 13 out. 2007.

WILSON, J. S; OTSUKI, T. Standards and Technical Regulations and Firms in Developing Countries: New Evidence from A World Bank Technical Barriers to Trade Survey. Washington: The World Bank, 2004a. 55p. Preliminary Draft.

WORLD TRADE ORGANIZATION. World trade report 2005. Disponível em: $<$ http://www.wto.org/english/news_e/pres05_e/pr4ll_e.htm>. Acesso em: 12 set. 2006.

ZHANG, H. P. Classification trees for multiple binary responses. Journal of the American Statistical Association, Boston, v. 93, p. 180-193, 1998. 